\title{
Management of arrythmia in the COVID 19 era
}

\author{
Tomos Sirma, Schröder Verginica, Margaritti Doina, Busuricu Florica \\ Faculty of Pharmacy, "Ovidius” University of Constanta, Romania \\ Email: sirmitomos@yahoo.com
}

\begin{abstract}
There is currently well-documented evidence that SARS CoV2 infection is frequently associated with secondary cardiac damage. Although lung damage is a priority, the evolution over time of COVID 19 infection frequently includes cardiovascular complications, the most common being: myocarditis, heart failure and acute coronary syndrome resulting from coronary artery thrombosis or plaque ruptures associated with SARS-CoV-2. Therefore, for the judicious use of resources, both human and material, and for the protection of medical staff, it is necessary to establish standard procedures to limit the contact of medical staff with the infected patient COVID 19.
\end{abstract}

Keywords. COVID 19, Arrhythmias, Severe acute respiratory syndrome, Viral infection, Atrial fibrillation, QT prolongation, Torsade de pointes

\section{Introduction}

The infection with the new Coronavirus 19 developed rapidly, to a global pandemic point, affecting, according to Johns Hopkins COVID-19 Resource Center, a number of 260,115,891 people between December 2019 and November 26, 2021 [1].

Currently, SARS-CoV2 puts us in front of some borderline situations, previously unknown, both from an epidemiological point of view and from a clinical point of view, situations in which we must associate the viral infection with the multiorgan damage that the new coronavirus develops, especially if we refer to the cytokine storm secondary to SARS CoV2 injection.

In this context, heart damage has a decisive impact in terms of the evolution of viral infection over time but also in terms of mortality rate.

Taking into account the population at risk of viral infection with COVID 19 and the population at high cardiovascular risk, we observe that they are superimposable, in the sense that in both situations the patients at risk are those over 60 years old, male, obese, diabetic or affected. anterior cardiovascular.

\section{Viral infection and cardiac damage}

\subsection{Cardiac arrhythmias}

There is currently well-documented evidence that SARS CoV2 infection is frequently associated with secondary cardiac damage. Although lung damage is a priority, the evolution over time of COVID 19 
infection frequently includes cardiovascular complications, the most common being: myocarditis, heart failure and acute coronary syndrome resulting from coronary artery thrombosis or plaque ruptures associated with SARS-CoV-2 [2].

Also, cardiac arrhythmias are frequently associated with viral infection with COVID 19. Thus, Liu et al. reported that approximately $7 \%$ of patients report palpitations as the only or first symptom of presentation [3], and physicians in Wuhan, China, reported that $16.7 \%$ of hospitalized patients and 44.4\% of ICU patients with COVID-19 had cardiac arrhythmias [4].

Recent studies have suggested that myocardial injury is particularly common in critically ill patients with COVID-19 through various mechanisms, mainly due to direct damage to cardiomyocytes and systemic inflammation.

The host-virus interaction leads to an alteration of the intercellular coupling, with the appearance of interstitial edema and cardiac fibrosis, with dysfunctions of the myocardial ion channels ( $\mathrm{Ca}$ and $\mathrm{K}$ channels) that will lead to conduction abnormalities and repolarization of the action potential. [5].

Not only the current COVID 19 virus has these characteristics. There are more than 20 viruses that have been implicated in myocardial inflammation and myocarditis, the most common being parvovirus B19, influenza virus, human immunodeficiency virus (HIV), Epstein-Barr virus, adenovirus and coxsackie virus B3 and do not occur only in subjects humans and animals, in vivo studies in SARS$\mathrm{CoV}$-infected mice and rabbits demonstrating direct inclusion of viral RNA in cardiomyocytes and impaired myocardial conduction system [6].

In a study by Sardana et al., over 17 million people with HIV were followed for a median period of 4.7 years and they found that people with HIV were at an increased risk of developing atrial fibrillation $\mathrm{AF}$ with a hazard ratio of 1.46 after adjusting for race, age, gender, socio-economic status, obesity, etc.

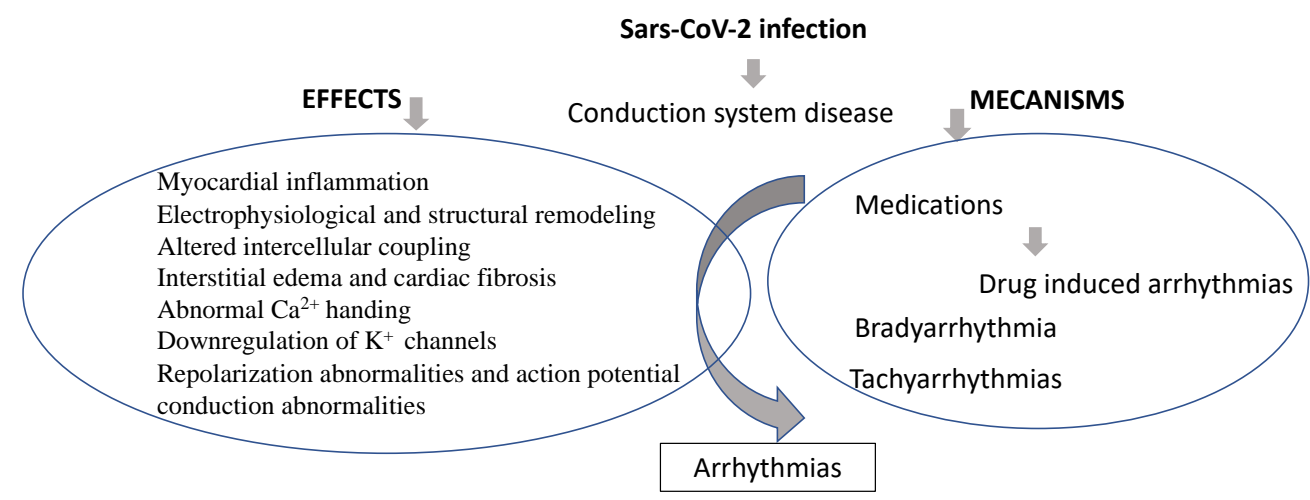

Figure 1 Sars-CoV-2 infection and arrhythmias possible mechanisms

Cardiac arrhythmias, more common in critically ill patients with SARS-CoV-2 (Figure 1), substrate hypoxia caused by direct lung damage that may be associated with myocarditis, abnormal host immune response, electrolyte dysfunction, myocardial ischemia, intravascular volume imbalances and finally side effects of antiviral treatment.

From the cardiac excitoconductive system the most often affected by viral infections are the sinus node and the atrio-ventricular node. According to Liu et al., 2001, the myocarditis process has three phases: phase one, viral infection, the entry of the virus and proliferation in the myocardium that may lead to the second phase (autoimmune phase) with $\mathrm{T}$ cell activation, cytokine production, and crossreacting antibodies formation and ultimately lead to phase 3 , cardiac remodelling and progressive cardiac dilation [7].

Clinically, myocarditis and pericarditis of viral etiology generally have reduced symptoms, being self-limiting. However, there are cases of ventricular tachycardia with a low prognosis secondary to viral myocardial damage [8].

Between March 27 and April 13, 2020, the Heart Rhythm Society (HRS) conducted a worldwide study, sending an online questionnaire to health professionals in 76 countries. The results of this study 
showed that the most common tachyarrhythmia associated with COVID 19 is atrial fibrillation, while the most common bradyarrhythmia were severe sinus bradycardia and complete atrio-ventricular block.

Ventricular tachycardia or ventricular fibrillation was found in $4.8 \%$ of respondents, amiodarone being the most commonly used medication for these situations [9].

This global survey of more than 1,100 respondents once again demonstrated the presence of arrhythmic manifestations, from benign to life-threatening, as side effects often associated with hydroxychloroquine and azithromycin therapy, which led to QT prolongation. on the electrocardiogram, which forced the discontinuation of treatment with these drugs.

Atrial fibrillation is the most common arrhythmia in patients with SARS-CoV2 infection, regardless of whether or not there is a previous cardiovascular disease.

Li Z. and Shao W. corroborated the data of 19 observational studies, which included 21,653 patients hospitalized with COVID-19, which showed that the prevalence of atrial fibrillation (AF) was $11 \%$, regardless of whether it was newly diagnosed or if patients had previously experienced paroxysmal AF [10].

The same meta-analysis showed that patients aged $>60$ years had a 2.5 times higher prevalence of AF compared to those $<60$ years (13 vs 5\%). The highest prevalence occurred in Europeans $(15 \%)$, followed by Americans (11\%), Asians (6\%) and Africans (2\%). In patients with severe COVID infection 19 the prevalence of AF was 6 times higher. compared to asymptomatic or minor symptoms (19 vs. $3 \%)$.

Moreover, the association of AF with SARS-CoV-2 has led to a significant increase in mortality in these patients, regardless of the cause. Mountantonakis S.E. and Saleh M. conducted a study of 9,564 patients hospitalized in New York with or without previously diagnosed AF, but who developed it during hospitalization. This study showed that hospital mortality was higher in patients with AF-54 compared to $37 \%$ (relative risk [RR)] 1.46, 95\% CI 1.34-1.59), in those with new AF the mortality rate appeared being even higher compared to those who already had $\mathrm{AF}(55.2 \%$ vs $46.8 \%)$, the deaths appearing after the hospitalization period [11].

Palpitations (symptomatic but not electrocardiographically documented) were reported in $7.3 \%$ of cases of SARS CoV-2 infection, according to a cohort study conducted in Hubei Province. In hospitalized patients they were reported at a percentage of $17 \%$ of the general cohort, and in those in intensive care at a percentage of $44 \%$ [12].

Long QT syndrome - due to dyselectrolyemia caused by cirrhosis infection COVID 19 has occurred frequently in hospitalized patients. Thus, a multicenter study conducted in 12 hospitals in New York City, Long Island, and Westchester County, New York, within the Northwell Health system shows that $6.1 \%$ of patients with SARS -CoV-2 infection had QT> 500 milliseconds at admission [13].

Also, therapy with hydroxychloroquine and azithromycin may increase the duration of the QT segment EKG> 435 milliseconds [14]. Various studies show that in most patients treated with one or both drugs there is a moderate increase in the QT interval, but the answer is individual reason why performing the electrocardiogram and electrolyte dosing before taking this medication is mandatory to properly assess the risk / benefit balance $[15,16]$.

In general, patients with the following QTc intervals have a low risk of significant QT prolongation and polymorphic TV in the course of the disease:

- QTc $<460$ milliseconds in men / women at puberty

- QTc $<470$ milliseconds in men after puberty

- QTc $<480$ milliseconds in women after puberty

If two to three hours after a dose of the drug with the potential to prolong the QT interval (such as hydroxychloroquine and / or azithromycin) the QTc increases to $\geq 500$ milliseconds or if the change in QT interval is $\geq 60$ milliseconds, a risk / benefit reassessment of the medicine, and set up the following steps:

- Discontinuation of all other medicines that prolong the QT interval.

- Correction of all electrolyte anomalies.

- Place the patient on continuous telemetry, considering a portable defibrillator or placement of external defibrillator patches. 
Another protocol proposes an ECG at baseline and again four hours after administration of QTprolonging medication in patients with congenital or acquired long QT syndrome, are already taking other drugs that prolong QT, or have structural heart disease or bradycardia [17].

There was a developed risk score in hospitalized patients for the prediction of QT prolongation, although not specifically for patients with COVID-19 [18]. It classifies patients as low, moderate, or high risk, which may allow triage if continuous telemetry is required during the use of QT prolongation drugs, especially if there are limited resources and the patient could be followed up on an outpatient basis $[18,19]$.

Bradyarrhythmia, including sinus pauses or high-grade heart block with slow escape rhythms, have not typically been seen.

Ventricular tachycardia, especially "torsades de pointes" has a serious prognosis, in these situations the patient requires an immediate and constant assessment (continuous monitoring) of symptoms, vital functions and level of consciousness because they can quickly and without warning become hemodynamically unstable.

Brugada Syndrome, because there is an increased risk of ventricular arrhythmias in fever in those with Brugada syndrome, aggressive reduction of acetaminophen fever is imperative. High-risk patients, such as those with spontaneous type 1 ECG and previous syncope, may consider going to an emergency department if they have a fever that cannot be rapidly reduced with acetaminophen [17].

As we observe, for the diagnostic and therapeutic management of patients with SARS-CoV-2 infection, regardless of the presence or not of the previous cardiovascular pathology, the frequent heart rhythm disorders occurred especially in patients "at risk" require both electrocardiographic monitoring and adjustment of antiviral therapy according to the electrocardiographic and electrolytic constants of the individually evaluated patient.

2.2. The patients with SARS-CoV2 infection with cardiovascular risk factors management For the judicious use of resources, both human and material, and for the protection of medical staff, it is necessary to establish standard procedures to limit the contact of medical staff with the infected patient COVID 19.

Therefore:

- Electrocardiogram at hospitalization, an EKG (preferably with 12 leads) will be performed to have documented the morphology of the electrocardiographic waves for the situations in which the patient develops symptoms that may suggest an acute coronary syndrome, as well as for documenting the QT interval. During hospitalization, if medication is used that prolongs this interval (hydroxychloroquine and / or azithromycin) patients should be monitored electrocardiographically, so as to reduce the possibility of acquired QT Syndrome.

- 24-hour Holter EKG monitoring - is necessary if there is a suspicion of cardiac ischemic damage or if there are pre-existing arrhythmias (medically documented). However, to reduce the impact of COVID 19 infection on the health system the number of staff interacting with the infected patient, with an increase in reaction time in critical situations) in many Western hospitals prefer continuous ECG monitoring vs. standard monitoring of vital functions by care staff.

- Thus, the patient's benefit is increased, the average medical staff is protected from infection and the costs of protective equipment are reduced.

- Transthoracic cardiac ultrasound is not usually performed in patients with SARS-CoV2 infection. It will be performed in case of symptoms specific to myocardial ischemia.

- In order to limit the exposure of the medical staff, the inpatient consultation of the patient with SARS-CoV2 will be performed by the infectionist doctor and at most by a cardiologist (in case of cardiac disorders that require it).

- During the hospitalization, consultations will be performed daily by a single member of the primary care team, the rest of the team participating from outside the room by video chat or telephone. 
- In stable patients with documented EKG arrhythmias or driving disorders, routine consultations will be performed without entering the patient's room, by analyzing previous EKG recordings, compared to Holter continuous monitoring data.

- If it is not urgent, for the same reasons to minimize the exposure of medical staff, cardiac consultations will be delayed in asymptomatic patients or with minor symptoms of SARS-CoV2 infection.

- If the patient's benefits exceed the risks of medical exposure and use of resources, both investigations and emergency treatment will be performed.

\subsubsection{The category of cardiovascular emergency procedures in patients with SARS-CoV2 infection includes:}

- TV ablation for uncontrolled electrical storm in hemodynamically unstable patients.

- Catheter ablation and / or cardioversion of symptomatic tachycardia, with significant hemodynamic changes, such as atrial fibrillation or atrial flutter that did not respond to antiarrhythmic drug treatment,

- Catheter ablation for Wolff-Parkinson-White syndrome or Atrial Fibrillation with syncope or cardio-respiratory arrest.

- Examination of the probe may be defective in a patient dependent on a pacemaker or in a patient with an implantable defibrillator who is receiving inappropriate therapy.

- Generator modification in pacemaker-dependent patients who are at the elective replacement indicator or at the end of the life of the device.

- Change the pacemaker or generator if the battery is low.

- Implantation of pacemaker for secondary prevention.

- Pacemaker implant for complete heart block, Mobitz II AV block or high-grade AV block with symptoms or severe symptomatic dysfunction of the sinus node.

- Cardiac resynchronization therapy in severe refractory heart failure in patients indicated in the guide.

- Cardioversion for atrial arrhythmias but exacerbated symptoms or with rapid uncontrolled rapid ventricular rates.

- Transesophageal echocardiogram for patients in urgent need of cardioversion.

- Basic life support and advanced cardiac life support for patients with COVID-19 should be administered as standard, similar to patients without COVID-19.

\section{Conclusions}

Cardiac arrhythmias, more common in critically ill patients with SARS-CoV2, substrate hypoxia caused by direct lung damage that may be associated with myocarditis, electrolyte dysfunction, myocardial ischemia.

In the diagnostic management of the patient with SARS-CoV2 infection with cardiovascular risk factors or of the patient associated with a heart disease (medically documented), it is necessary to perform tests both at hospitalization and during hospitalization for viral infection.

\section{References}

[1] COVID-19 Map. In: Johns Hopkins Coronavirus Resource Center. Available: https://coronavirus.jhu.edu/map.html. Accessed 26.November 2021

[2] S. BABAPOOR-FARROKHRAN, D. GILL, J. WALKER, R.T. RASEKHI, B. BOZORGNIA, A. AMANULLAH: Myocardial injury and COVID-19: possible mechanisms. Life Sci. 2020; 253 :117723. doi: 10.1016/j.lfs.2020.117723.

[3] K. LIU, Y.Y. FANG, Y. DENG, W. LIU, M.F.WANG, J.P. MA, W. XIAO, Y.N. WANG, M.H. ZHONG, CH. LI, GC. LI, HG. LIU: Clinical characteristics of novel coronavirus cases in 
tertiary hospitals in Hubei Province. Chin Med J. 2020;133 (9):10251031. doi:10.1097/CM9.0000000000000744

[4] D. WANG, B. HU, C. HU, F. ZHU, X. LIU, J. ZHANG, B. WANG, H. XIANG, Z. CHENG, Y. XIONG, Y. ZHAO, Y. LI, X. WANG, Z. PENG:Clinical Characteristics of 138 Hospitalized Patients With 2019 Novel Coronavirus-Infected Pneumonia in Wuhan, China. JAMA. 2020;323(11):1061-1069. doi:10.1001/jama.2020.1585

[5] G. TSE, J.M. YEO, Y.W. CHAN, LAI ETHL, B.P. YAN: What Is the Arrhythmic Substrate in Viral Myocarditis? Insights from Clinical and Animal Studies Front Physiol. 2016; 7 :308. doi: 10.3389/fphys.2016.00308.

[6] L. K. ALEXANDER, B. W. KEENE, J. D. SMALL, B. YOUNT, R. S. BARIC. Electrocardiographic changes following rabbit coronavirus-induced myocarditis and dilated cardiomyopathy. Adv Exp Med Biol.1993;342:365-370. doi:10.1007/978-1-4615-2996-5_56.

[7] P. P. LIU, J.W. MASON: Advances in the understanding of myocarditis. Circulation. 2001;104 (9): 1076-1082. doi: 10.1161/hc3401.095198

[8] A. J. BAKSI, G. S. KANAGANAYAGAM, S. K. PRASAD: Arrhythmias in viral myocarditis and pericarditis. Card Electrophysiol Clin. 2015;7(2):269-281. doi:10.1016/j.ccep.2015.03.009 - 10.1007/s10840-020-00789-9

[9] Z. LI, W. SHAO, J. ZHANG, J. MA, S. HUANG, P. YU, W. ZHU, X. LIU: Prevalence of Atrial Fibrillation and Associated Mortality Among Hospitalized Patients With COVID-19: A Systematic Review and Meta-Analysis. Front Cardiovasc Med. 2021;8:720129. Epub 2021 Oct 13; https://doi.org/10.3389/fcvm.2021.720129

[10] S. E. MOUNTANTONAKIS, M. SALEH, J. FISHBEIN, A. GANDOMI, M. LESSER, J. CHELICO, J. GABRIELS, M. QIU, L. M. EPSTEIN: Atrial fibrillation is an independent predictor for in-hospital mortality in patients admitted with SARS-CoV-2 infection. Northwell COVID-19 Research Consortium Heart Rhythm. 2021;18(4):501-507; doi: 10.1016/j.hrthm.2021.01.018. Epub 2021 Jan 22.

[11] T. GUO, Y. FAN, M. CHEN, X. WU, L. ZHANG, T. HE, H. WANG, J. WAN, X. WANG, Z. LU:Cardiovascular Implications of Fatal Outcomes of Patients With Coronavirus Disease 2019 (COVID-19) - JAMA Cardiol. 2020;5(7):811-818; DOI: 10.1001/jamacardio.2020.1017

[12] S. RICHARDSON, J. S. HIRSCH, M. NARASIMHAN, J. M. CRAWFORD, T. MCGINN, K. W. DAVIDSON et. al.:The Northwell COVID-19 Reasearch Consortium: Presenting Characteristics, Comorbidities, and Outcomes Among 5700 Patients Hospitalized With COVID-19 in the New York City Area. JAMA. 2020;323(20):2052-2059; https://doi.org/10.1001/jama.2020.6775

[13] E. CHORIN, M. DAI, E. SHULMAN, A. L. ET:The QT interval in patients with COVID-19 treated with hydroxychloroquine and azithromycin. Nat Med. 2020; 26, 808-809; https://doi.org/10.1038/s41591-020-0888-2

[14] A. MAZZANTI, M. BRIANI, D. KUKAVICA, F. BULIAN:Association of Hydroxychloroquine With QTc Interval in Patients With COVID-19. Circulation. 2020;142(5):513. https://doi.org/10.1161/CIRCULATIONAHA.120.048476

[15] O. VOISIN, E. L. LORC'H, MAHÉA: Acute QT Interval Modifications During Hydroxychloroquine-Azithromycin Treatment in the Context of COVID-19 Infection. Mayo Clin Proc. 2020;95 (8):1696. https://dx.doi.org/10.1016\%2Fj.mayocp.2020.05.005

[16] C. I. WU, P. G. POSTEMA, E. ARBELO, E. R. BEHR: SARS-CoV-2, COVID-19, and inherited $\begin{array}{llll}\text { arrhythmia } \quad \text { Syndromes. } & \text { Heart 2020;17(9):1456. }\end{array}$ https://doi.org/10.1016/j.hrthm.2020.03.024

[17] J. E. TISDALE, H. A. JAYNES, J. R. KINGERY, N. A. MOURAD: Development and validation of a risk score to predict QT interval prolongation in hospitalized pacients - Cardiovasc Qual Outcomes. 2013;6 (4):479. https://doi.org/10.1161/CIRCOUTCOMES.113.000152

[18] https://www.acc.org/latest-in-cardiology/articles/2020/03/27/14/00/ventricular-arrhythmia-riskdue-to-hydroxychloroquine-azithromycin-treatment-for-covid-19 